\title{
EXPEDIÇÃO BOTANICA URBANA EM SETE CIDADES COLOMBIANAS
}

Luis Fernando Molina-Prieto ${ }^{1}$

\section{RESUMO}

As arborizações urbanas na Colômbia e em outros países, geram grandes benefícios ambientais para as cidades, mas também são fonte de inúmeros problemas ambientais, econômicos e de saúde pública. O artigo apresenta os resultados obtidos em sete cidades do país (Bucaramanga, Cúcuta, Ibagué, Neiva, Palmira, Popayán e Villavicencio), para as quais foram determinadas: i) as espécies com populações representativas de cada cidade, ii) as espécies impróprias para arborização urbana, e iii) as espécies recomendadas pela pesquisa, pois além de ser adequadas para áreas urbanas, eles contribuem para o fortalecimento da Estrutura Ecológica Principal em quatro dos seus componentes: solo, água, fauna e ecossistemas.

Palavras-chave: Arborização urbana; Estrutura Ecológica Principal.

\section{URBAN BOTANICAL EXPEDITION IN SEVEN COLOMBIAN CITIES}

\section{ABSTRACT}

Urban tree planting in Colombia and other countries has produced great ecological benefits to human settlements. However, they have brought along countless environmental, economic and public health problems as well. The article presents the results of a comparative study conducted in seven mid-sized cities (Bucaramanga, Cucuta, Ibague, Neiva, Palmira, Popayan and Villavicencio), narrowed down to three specific data: i) the most-representative tree species per city, ii) the nonrecommended species for urban tree planting, and iii) the highly recommended species for urbanized areas, essentially, those strengthening the Main Ecological Structure on its four principal components: soil, water, fauna and ecosystem.

Keywords: Urban tree planting; Ecological Principal Structure.

\footnotetext{
${ }^{1}$ Arquitecto-Investigador de la Facultad de Artes, Universidad Antonio Nariño, Bogotá, Colombia, e-MAIL: molinaprieto@yahoo.com.ar 
Las arborizaciones urbanas son esenciales para la sostenibilidad de las ciudades, pues desempeñan una serie muy amplia de funciones que ningún otro elemento urbano puede generar. Los árboles plantados en parques, calles y rondas hídricas, aportan a las ciudades beneficios de diversa índole como veremos a continuación a) Ecológicos: producción de alimento y disposición de hábitat para las aves urbanas tanto nativas como migratorias, lo que contribuye con su conservación; control de la erosión, estabilización de taludes y enriquecimiento (nitrificación) del suelo, lo que evita deslaves o remociones en masa y contribuye a la fertilidad del suelo urbano; protección de cuencas, microcuencas urbanas y cuerpos de agua, conservación de rondas hídricas y regulación de los caudales, lo que reduce los riesgos de desastre en épocas de invierno o alta pluviosidad; b) Ambientales: mejoramiento de la calidad del aire, captación de partículas en suspensión; captación de dióxido de carbono, regulación climática y control de la temperatura, reducción del impacto del viento, reducción de niveles de ruido, lo que hace más habitables y confortables los espacios públicos; c) Paisajísticos: mejoramiento de la calidad estética de las áreas urbanas, conformación y caracterización de espacios urbanos, lo que enriquece el paisaje urbano. d) Psicológicos. Aporte cultural simbólico, aporte al bienestar psicológico y la recreación, lo que fomenta habitantes más saludables y satisfechos. e) Económicos. Valorización de la propiedad, producción de frutos para consumo humano, empleo e ingreso generado por el mantenimiento de las arborizaciones urbanas (WIESNER, 2000, p. 21-25; RODRIGUES; CARVALHO, 2010, p. 44).

De otro lado hay que tener en cuenta los daños generados por los árboles en los espacios urbanos, resultado de plantar las especies inapropiadas para las ciudades (especies con raíces agresivas por ejemplo), o por plantar especies en espacios urbanos inadecuados para ellas (árboles de gran porte bajo tendidos eléctricos, por ejemplo). Estos daños igualmente son de diversa índole a) Ecológicos: carencia de oferta alimenticia para las aves urbanas tanto nativas como migratorias, lo que contribuye con su extinción; erosión del suelo, desestabilización de taludes y pérdida de nutrientes del suelo, lo que genera deslaves y remociones en masa y contribuye a la pérdida de fertilidad del suelo urbano; desecamiento de cuencas, microcuencas urbanas y cuerpos de agua, afectación de rondas hídricas y disturbio de los caudales, lo que incrementa los riesgos de desastre en épocas de invierno o alta pluviosidad; b) Económicos: desvalorización de la propiedad; gastos de reparación de tuberías de aguas servidas, asfaltos, andenes, obras arquitectónicas y civiles (por raíces agresivas); gastos de mantenimiento (podas continuas) a árboles plantados de manera inapropiada que afectan redes aéreas de telefonía, electricidad y televisión; impacto al tráfico automotor durante las reparaciones a daños causados bajo las vías vehiculares o en su entorno; y daños materiales a bienes particulares como automóviles y viviendas que son destruidos parcial o totalmente por el desplome de grandes árboles o caída de ramas de especies que se autopodan (riesgos que las compañías de seguros no cobijan en Colombia); c) Psicológicos y sanitarios: daños físicos y psicológicos debido a accidentes en el espacio urbano, en muchos casos fatales, generados por la caída de grandes frutos, enormes ramas o árboles completos, y afectaciones leves o severas a la salud humana por causa de especies tóxicas.

Los árboles urbanos poseen grandes potenciales para el mejoramiento de la calidad de vida de los habitantes de las ciudades, y contribuyen a la sostenibilidad de las mismas, siempre y cuando se planten las especies adecuadas en los espacios que les permiten desarrollarse en toda su plenitud. Pero como hemos visto, los árboles urbanos también pueden generar grandes daños, de manera que es necesario y urgente trazar políticas claras en relación a la arborización urbana, políticas que deben incluir, especialmente, las especies que por sus características físico-químicas sean apropiadas para los centros urbanos (y los lugares adecuados para plantarlas), así como las que no se deben plantar porque causan daños a las construcciones y/o afectaciones a la salud humana. 


\section{Estudios sobre arborización urbana}

Cinco ciudades colombianas cuentan con libros que describen las especies plantadas en sus espacios públicos: Cali, Cúcuta (inédito), Bogotá, Medellín y Leticia. Se trata de estudios organizados por medio de fichas que presentan los aspectos físicos y botánicos de cada especie, como son: nombre científico y nombres comunes; origen de la especie; descripción de tamaño (en pocos casos del diámetro de la copa), descripción y/o fotografías de hojas, follaje, flores y frutos; sistemas de propagación y funciones paisajísticas. Todas las publicaciones coinciden en el apartado usos, el cual se refiere al uso que se le puede dar a la madera de las distintas especies, lo que no es relevante para los procesos de arborización urbana: "Su madera es dura y resistente al ataque del comején, sirve para fabricar vigas, postes y cercas” (HERRERA, 2009, p. 52), “Su madera es utilizada en la elaboración de mangos para herramientas" (CÁRDENAS et al, 2004, p. 77); o al uso que se le da a la especie fuera de las ciudades, que tampoco es un dato pertinente: "Comúnmente ha sido utilizado para sombrío en cultivos de café y cacao" (BARÓN; MORALES, 1997, p. 87), "Se emplea en algunos cultivos como sombrío” (SECRETARÍA DEL MEDIO AMIENTE/JARDÍN BOTÁNICO DE MEDELLÍN, 2007, p. 140); además, claro está, de los usos como especie ornamental en las ciudades. Pero sólo en contadas excepciones se alude a los beneficios ecológicos de cada especie, es decir, cuáles recuperan suelos erosionados, cuáles conservan cuencas o rondas hídricas, y cuáles alimentan la avifauna urbana.

En cuanto a las especies caracterizadas por sus raíces agresivas, la ambigüedad es lo más común en estos estudios, puesto que aunque los autores reportan la agresividad de las mismas, no descartan tales especies de la arborización urbana:

“La raíz de este árbol es muy gruesa y superficial, por lo cual afecta las edificaciones vecinas [...] Sin embargo, puede podarse la raíz durante la estación seca [...] Debe alejarse de las construcciones a una distancia prudencial de 20 m.” (CALDAS, 1975, P. 83).
"Sistema radicular superficial, sus raíces laterales son extensas, hay reportes de raíces laterales de 150 metros de largo (HERRERA, 2009, p. 37).

Este tipo de información no es clara ni útil, especialmente para quienes consultan estos estudios con la intención de seleccionar las especies para la arborización urbana (arquitectos, urbanistas y planificadores urbanos, por lo general poco o nada relacionados con la Botánica), puesto que los autores no aclaran cuáles especies pueden causar daños a las obras arquitectónicas y urbanas, y cuáles no las afectarán a mediano o largo plazo. Si bien estas publicaciones han colaborado en gran medida al conocimiento de las especies que se encuentran en las ciudades mencionadas, no han impedido que se sigan plantando especies inadecuadas, que generan, como ya se dijo, daños severos a la infraestructura vial y de servicios públicos; daños a vehículos, construcciones arquitectónicas y civiles, e incluso, afectaciones a la salud y la vida de algunos ciudadanos.

Dos ciudades colombianas son la excepción: Bogotá y Medellín, puesto que en ellas las autoridades ambientales, las alcaldías y los jardines botánicos locales, han trabajado conjuntamente para elaborar manuales técnicos y otras publicaciones, que trazan políticas claras en relación a las arborizaciones urbanas, al tiempo que recomiendan un grupo de especies a plantar (las adecuadas), y dejan por fuera muchas otras (las inadecuadas). Cabe destacar, para Bogotá el Manual Verde (JARDÍN BOTÁNICO DE BOGOTÁ, 1999) que recomienda 15 especies; la Guía para la plantación de árboles (DAMA-CONIF, 1999), que recomienda 8 especies; el libro Especies ornamentales usadas en áreas urbanas "Experiencia programa hojas verdes" (DELGADO, 1995) que recomienda 33 especies; y para Medellín el Manual de silvicultura urbana para Medellín (SECRETARÍA DEL MEDIO AMIENTE/JARDÍN BOTÁNICO DE MEDELLÍN, 2007) que recomienda 100 especies. Pero en el resto de los casi mil municipios colombianos, no se cuenta con este tipo de políticas, ni siquiera en las ciudades intermedias ni en las capitales de departamento. 
Teniendo en cuenta todo lo anterior se adelantó una investigación en siete ciudades colombianas: Cúcuta, Bucaramanga, Ibagué, Neiva, Palmira, Popayán y Villavicencio, que buscó dar respuesta a tres grandes interrogantes: ¿Cuáles especies conforman la arborización urbana de cada una de esas ciudades?, ¿Cuáles especies son inapropiadas o no aptas para los espacios urbanos por los riesgos y daños que generan? y ¿cuáles especies, por sus características particulares, fortalecen la Estructura Ecológica Principal EEP² de cada una de esas ciudades?

\section{MATERIALES Y MÉTODOS}

\section{Caracterización del área de estudio}

Las ciudades incluidas en el estudio se ubican en distintas regiones del país y cuentan con características particulares. Bucaramanga: capital del departamento de Santander, se localiza en la Cordillera Oriental de los Andes colombianos al nordeste del país. Altitud 959 msnm. Temperatura promedio: 24 grados centígrados. Cúcuta: capital del departamento de Norte de Santander, se localiza en la Cordillera Oriental de los Andes colombianos al nordeste del país, en la frontera con Venezuela. Altitud 320 msnm. Temperatura promedio: 28 grados centígrados. Ibagué: Capital del departamento del Tolima, se localiza en la Cordillera Central de los Andes colombianos en la zona central del país. Altitud: 1285 msnm. Temperatura promedio: 24 grados centígrados. Neiva: Capital del departamento del Huila, se localiza entre las Cordilleras Central y Oriental de los Andes colombianos, en una planicie sobre la margen oriental del río Magdalena, en la zona centro-sur del país. Altitud: 442 msnm. Temperatura promedio: de 28 a 37 grados centígrados (de acuerdo a la época del año). Palmira: municipio del departamento del Valle del Cauca, se localiza en la Cordillera Central de los Andes colombianos en el suroriente del país. Altitud: 1001 msnm. Temperatura promedio: 23 grados centígrados.

\footnotetext{
${ }^{2}$ La Estructura Ecológica Principal es un concepto que rige los Planes de Ordenamiento Territorial en Colombia, y está conformada por las áreas de reserva ambiental, los parques y las rondas hídricas dentro y entorno al perímetro urbano.
}

Popayán: capital del departamento del Cauca, se ubica sobre la Cordillera de los Andes, al suroccidente del país. Altitud: 1740 msnm. Temperatura promedio: de 19 grados centígrados. Villavicencio: capital del departamento del Meta, se ubica en los Llanos Orientales, en el piedemonte de la Cordillera Oriental de los Andes colombianos. Altitud: 467 msnm. Temperatura promedio: de 28 grados centígrados.

\section{Metodología aplicada}

La metodología abarcó tres etapas.

-En primer lugar se establecieron las especies de árboles que cuentan con poblaciones representativas ${ }^{3}$ en cada una de las ciudades estudiadas. Para esto se contó con auxiliares de investigación (profesores de la Universidad Antonio Nariño) residentes en cada una de las ciudades que abarcó el estudio, que adelantaron la revisión de documentos relacionados con los objetivos de la investigación, expedidos por entidades públicas o privadas locales. Paralelamente se realizó la revisión bibliográfica, y se adelantaron consultas vía Internet con los jardines botánicos y con las autoridades ambientales locales (Alcaldías, Corporaciones Autónomas Regionales) de cada ciudad. Luego se realizaron labores en las áreas de estudio, se visitaron las entidades locales, se consultaron expertos en el tema y se realizaron los trabajos de campo (ocho días por ciudad), con el fin de establecer el listado definitivo de especies empleadas en la arborización urbana de cada ciudad. Además, se tomaron fotografías de cada especie, y de cada uno de sus elementos principales: hojas, frutos, flores, corteza, tronco, y base del tronco (para registrar los aspectos superficiales de la raíz).

-En segundo lugar, a partir de los listados de especies establecidos, se consultaron diversos referentes bibliográficos y páginas web especializadas en el tema en

\footnotetext{
${ }^{3}$ Con poblaciones representativas nos referimos a especies que se encontraron en diversas áreas de la ciudad, y en número o cantidad tal, que permitió inferir su empleo en programas de arborización urbana. De modo que no se tuvieron en cuenta especies que contaban con escaso número de individuos en la ciudad, como por ejemplo el corcho Quercus suber, en Popayán, del cual sólo se cuentan dos ejemplares en el parque central.
} 
busca de datos técnicos, se cotejaron con los datos obtenidos en los trabajos de campo, y así se estableció un listado con las especies inapropiadas para arborizaciones urbanas, por ser fuentes potenciales de daños a infraestructuras y/o riesgos a la salud humana.

Finalmente, partir de las mismas fuentes referenciales y los datos obtenidos en los trabajos de campo, se seleccionaron las especies de árboles que contribuyen al fortalecimiento de la Estructura Ecológica Principal-EEP de cada una de las ciudades objeto de estudio. Para esto se creó una matriz con cuatro componentes básicos de la EEP: suelo, agua, fauna y ecosistema, con un total de 18 variables (la matriz se presenta más adelante).

\section{RESULTADOS Y DISCUSIÓN}

\section{Especies con poblaciones representativas en siete} ciudades

Los listados por ciudad se establecieron con base en las siguientes fuentes. Bucaramanga: se consultó a expertos de la Corporación Autónoma Regional para la Defensa de la Meseta de Bucaramanga CDMB, y a profesionales del Jardín Botánico Eloy Valenzuela, se revisaron las fichas de árboles publicadas por el diario Vanguardia Liberal, y se complementó la información con el trabajo de campo (esta ciudad fue visitada en tres oportunidades). Cúcuta: se tomó como base el estudio Cúcuta verde y exuberante de Carvajal E. \& J. Chacón, publicado (en archivo digital) por la Corporación Autónoma Regional de la
Frontera Norte-CORPONOR en 1999; y se complementó y actualizó la información con el trabajo de campo. Ibagué: se realizaron consultas con expertos del Jardín Botánico Alexander von Humboldt de la Universidad del Tolima, se revisó el Estatuto del Árbol Urbano, documento expedido por el Consejo Municipal de Ibagué en 1989, y se complementó la información con el trabajo de campo. Neiva: se revisó el Acuerdo 044 de 2003, expedido por el Consejo de Neiva, que trata de "Las competencias en materia de arborización en el espacio público de la ciudad de Neiva”, y se complementó la información con el trabajo de campo. Palmira se revisó el documento Árboles, arbustos y aves en el agrosistema del CIAT (sede Palmira), y se complementó la información con el trabajo de campo. Popayán: se consultó a expertos de la Corporación Autónoma Regional del Cauca CRC, se revisaron estudios puntuales realizados por la CRC en algunos parques de la ciudad, y se complementó la información con el trabajo de campo.

Villavicencio: se revisó el documento Estudio diagnóstico y formulación de alternativas de solución a las afectaciones de la flora urbana sobre la infraestructura vial y de servicios públicos, y se complementó la información con el trabajo de campo.

En las Tablas 1 y 2 se presentan las especies incluidas en este estudio, y las ciudades en las que cuentan con poblaciones representativas. En la Tabla 1, las especies nativas; en la Tabla 2, las especies exóticas o introducidas. 
Tabla 1. Especies nativas en siete ciudades colombianas

Table 1. Native species in seven Colombian cities

\begin{tabular}{|c|c|c|c|c|c|c|c|}
\hline \multirow{2}{*}{ ESPECIES } & \multicolumn{7}{|c|}{ CIUDADES } \\
\hline & Buc. & Cúc. & Iba. & Nei. & Pal. & Pop. & Vill. \\
\hline Acacia forrajera Leucaena leucocephala & $\mathrm{X}$ & $\mathrm{X}$ & $\mathrm{X}$ & $\mathrm{X}$ & $\mathrm{x}$ & $\mathrm{X}$ & $\mathrm{X}$ \\
\hline Achiote Bixa orellana & & & $\mathrm{X}$ & $\bar{x}$ & & & \\
\hline Algarrobo Hymenaea courbaril & & & & & $\mathrm{x}$ & & $\mathrm{x}$ \\
\hline Aliso Alnus acuminata & & & & & & $\mathrm{X}$ & \\
\hline Aguacate Persea americana & $\mathrm{X}$ & & & & $\mathrm{X}$ & $\mathrm{X}$ & $\mathrm{X}$ \\
\hline Arrayán Myrcianthes leucoxila & & & & & & & $\bar{x}$ \\
\hline Bala de cañón Couropita gianensis & $\mathrm{x}$ & & & & $\mathrm{x}$ & & \\
\hline Balso Ocbroma pyramidalis & & & $\mathrm{X}$ & $x$ & & $\mathrm{x}$ & $x$ \\
\hline Búcaro Erytbrina fusca & $\mathrm{x}$ & $\mathrm{x}$ & $\bar{x}$ & $\bar{x}$ & $x$ & $\mathrm{X}$ & $\bar{x}$ \\
\hline Cacao Theobroma cacao & & & & $\bar{x}$ & & & $\bar{x}$ \\
\hline Carbonero Calliandra pitieri & & & $x$ & $\mathrm{x}$ & $x$ & $\mathrm{X}$ & \\
\hline Carbonero rojo Calliandria carbonaria & & & & & & $\mathrm{x}$ & \\
\hline Caucho sabanero Ficus soatensis & & & & & & $\mathrm{X}$ & \\
\hline Cachimbo Erytbrina poeppigiana & $\mathrm{X}$ & & $\mathrm{x}$ & & $\mathrm{x}$ & & $\mathrm{x}$ \\
\hline Chachafruto Erytbrina edulis & $x$ & & & & $\mathrm{x}$ & $\mathrm{x}$ & $\bar{x}$ \\
\hline Caracolí Anacardium excelsum & $\mathrm{x}$ & $\mathrm{X}$ & $x$ & $x$ & $\mathrm{x}$ & & $x$ \\
\hline Cedro Cedrela montana & & & & & & $\mathrm{X}$ & \\
\hline Ceiba Ceiba pentandra & $\mathrm{x}$ & $\mathrm{x}$ & $\bar{x}$ & $\bar{x}$ & $\mathrm{x}$ & $\mathrm{X}$ & $\bar{x}$ \\
\hline Cerezo capulí Prunus serotina & & & & & & & $x$ \\
\hline Chicalá Tecoma stans & $\mathrm{x}$ & $\mathrm{X}$ & $\mathrm{X}$ & $\mathrm{X}$ & $\mathrm{x}$ & $\mathrm{x}$ & $\mathrm{x}$ \\
\hline Coralito Cordia sebestana & & $\bar{x}$ & & & & & \\
\hline Cují Prosopis juliflora & & $\mathrm{x}$ & & $\bar{x}$ & & & \\
\hline Divi divi de tierra fría Caesalpinia spinosa & & & & & & & $\bar{x}$ \\
\hline Ébano Caesalpinia ebano & & & & & $\mathrm{X}$ & & \\
\hline Gallinero Pithecellobium dulce & $\mathrm{X}$ & $\mathrm{X}$ & $\bar{x}$ & $\bar{x}$ & $\mathrm{X}$ & & $\bar{x}$ \\
\hline Guadua Bambusa guadua & $\mathrm{X}$ & $\mathrm{x}$ & $\bar{x}$ & $\bar{x}$ & $\mathrm{X}$ & $\mathrm{X}$ & $\bar{x}$ \\
\hline Gualanday Jacaranda caucana & & & $\bar{x}$ & $\bar{x}$ & & $\mathrm{X}$ & \\
\hline Guásimo Guazuma ulmifolia & $\mathrm{X}$ & & & $\mathrm{X}$ & $\mathrm{X}$ & & $x$ \\
\hline Guamo Inga edulis & $\mathrm{x}$ & & $\bar{x}$ & & $\mathrm{X}$ & $\mathrm{X}$ & $\bar{x}$ \\
\hline Guayabo Psidium guajaba & $\mathrm{X}$ & & $\bar{x}$ & & $\mathrm{X}$ & $\mathrm{X}$ & $\bar{x}$ \\
\hline Guayacán de Manizales Lafoensia speciosa & & & & & & $\mathrm{X}$ & \\
\hline Guayacán flor amarillo Tabebuia chrysantha & $\mathrm{X}$ & $\mathrm{X}$ & $\bar{x}$ & $\bar{x}$ & $\mathrm{X}$ & $\mathrm{X}$ & $\bar{x}$ \\
\hline Guayacán rosado Tabebuia rosea & $\mathrm{x}$ & $\mathrm{X}$ & $\bar{x}$ & $\bar{x}$ & $\mathrm{X}$ & $\mathrm{X}$ & $\bar{x}$ \\
\hline Iguá Pseudosamanea guachapele & & & $\bar{x}$ & $\bar{x}$ & & & $\bar{x}$ \\
\hline Habillo Hura crepitans & $\mathrm{X}$ & $\mathrm{X}$ & $\bar{x}$ & $\bar{x}$ & $\mathrm{X}$ & $\mathrm{X}$ & $\bar{x}$ \\
\hline Jalapo Albizizia caronaria & $x$ & & & & & & \\
\hline Madroño Rheedia madruno & & & $\bar{x}$ & & & & \\
\hline Mamey Mammea americana & & & $\mathrm{x}$ & & & & \\
\hline Mamón Melicoccus bijugatus & $\mathrm{X}$ & $\mathrm{X}$ & $\bar{x}$ & $\bar{x}$ & $\mathrm{X}$ & & $\bar{x}$ \\
\hline Mano de tigre Sterculia apetala & $x$ & $x$ & & & $\mathrm{x}$ & & \\
\hline Matarratón Gliricidia sepium & $\mathrm{X}$ & $\mathrm{X}$ & & $\mathrm{x}$ & $\mathrm{X}$ & & $\bar{x}$ \\
\hline Mayo Meriania speciosa & & & & & & $\mathrm{X}$ & \\
\hline Nacedero Trichanthera gigantea & & & & & & $\mathrm{X}$ & \\
\hline Níspero Acbras zapota & & $\mathrm{X}$ & & & & & \\
\hline Nogal Juglans neotropica & & & & & & $\mathrm{X}$ & \\
\hline Orejero Enterolobium yyclocarpum & $\mathrm{x}$ & & & & & & \\
\hline Palma zancona Syagrus sancona & & & & & $\mathrm{x}$ & $\mathrm{x}$ & $\mathrm{x}$ \\
\hline Palo de cruz Brownea ariza & & & $x$ & $x$ & $\mathrm{x}$ & & $\bar{x}$ \\
\hline Pino romerón Decussocarpus rospigliosii & & & & & & $\mathrm{x}$ & \\
\hline Roble Quercus bumboldtii & & & & & & $\mathrm{x}$ & \\
\hline Samán Samanea saman & $\mathrm{x}$ & $\mathrm{x}$ & $x$ & $x$ & $\mathrm{x}$ & & $x$ \\
\hline Sapotolongo Pachira acuatica & $\mathrm{X}$ & $\mathrm{X}$ & & $x$ & $\mathrm{x}$ & & $\bar{x}$ \\
\hline Sarrapio Coumarouna odorata & $\mathrm{x}$ & & & & & & \\
\hline Sauce Salix humboldtiana & & & & & & $\mathrm{x}$ & \\
\hline Saúco Sambucus $S p$. & & & & & & & $\mathrm{x}$ \\
\hline Sietecueros Tebouchina lepidota & & & & & & & $\bar{x}$ \\
\hline Tambor Schizolobium parabybum & $\mathrm{x}$ & & & & & & \\
\hline Totumo Crecentia cujete & $\mathrm{x}$ & $\mathrm{X}$ & $\mathrm{X}$ & $x$ & $\mathrm{X}$ & & $\mathrm{X}$ \\
\hline Yarumo Cecropia sp. & $\mathrm{x}$ & & $\mathrm{X}$ & $\mathrm{x}$ & $\mathrm{x}$ & $\mathrm{x}$ & $\mathrm{x}$ \\
\hline Yopo Piptadenia peregrina & & & & & & & $\bar{x}$ \\
\hline Zapote Matisia cordata. & $\mathrm{x}$ & & & $\mathrm{x}$ & $\mathrm{x}$ & & $\mathrm{x}$ \\
\hline
\end{tabular}


Tabla 2. Especies exóticas en siete ciudades colombianas

Table 2. Exotic species in seven Colombian cities

\begin{tabular}{|c|c|c|c|c|c|c|c|}
\hline \multirow{2}{*}{ ESPECIES } & \multicolumn{7}{|c|}{ CIUDADES } \\
\hline & Buc. & Cúc. & Iba. & Nei. & Pal. & Pop. & Vill. \\
\hline Acacia amarilla Cassia siamea & $\mathrm{X}$ & $\mathrm{X}$ & $\mathrm{X}$ & $\mathrm{X}$ & $\mathrm{X}$ & & $\mathrm{X}$ \\
\hline Acacia de Girardot Delonix regia & $\mathrm{X}$ & $\mathrm{X}$ & $\mathrm{x}$ & $\mathrm{x}$ & $\mathrm{x}$ & & $\mathrm{x}$ \\
\hline Acacia japonesa Acacia melanoxylon & & & & & & $\mathrm{X}$ & \\
\hline Acacia rubiña Caesalpinia peltohoroides & & & & & $\mathrm{X}$ & $\mathrm{X}$ & \\
\hline Acacia sabanera Albiqia lophanta & & & & & & $\bar{x}$ & \\
\hline Almendro Terminalia catappa & $\mathrm{X}$ & $\mathrm{X}$ & $\mathrm{X}$ & $\mathrm{x}$ & $\mathrm{x}$ & & $\mathrm{X}$ \\
\hline Araucaria Araucaria excelsa & $\mathrm{X}$ & $\mathrm{X}$ & $\mathrm{X}$ & & $\mathrm{x}$ & $\bar{x}$ & $x$ \\
\hline Araucaria Araucana Araucaria araucana & & & & & & & $\bar{x}$ \\
\hline Árbol del pan Artocarpus altilis & $\mathrm{X}$ & $\mathrm{X}$ & & $\mathrm{X}$ & $\mathrm{x}$ & & $x$ \\
\hline Árbol nim Melia indica & & $\mathrm{X}$ & $\mathrm{X}$ & $\mathrm{X}$ & $\mathrm{x}$ & & $\mathrm{X}$ \\
\hline Candelabros Euphorbia lactea & & $\mathrm{X}$ & & & & & \\
\hline Carbonero blanco Calliandra abematocephala & $\mathrm{X}$ & $\mathrm{X}$ & & & $\mathrm{X}$ & $\bar{x}$ & $\bar{x}$ \\
\hline Cadmia Cananga odorata & & & & & $\mathrm{X}$ & & $\mathrm{X}$ \\
\hline Caucho benjamín Ficus benjamina & & & $\mathrm{X}$ & & & & \\
\hline Caucho de la India Ficus elastica & $\mathrm{X}$ & $\mathrm{X}$ & $\bar{x}$ & $\mathrm{x}$ & $\bar{x}$ & $\mathrm{x}$ & $\mathrm{X}$ \\
\hline Caucho lira Ficus lirata & $\bar{x}$ & $\mathrm{X}$ & & & $\mathrm{x}$ & & \\
\hline Casuarina Casuarina esiquetifolia & & & $\mathrm{X}$ & & & $\mathrm{X}$ & $\mathrm{X}$ \\
\hline Ceibo dominico Erythrina indica & $\mathrm{X}$ & $\mathrm{X}$ & $\mathrm{X}$ & & $\mathrm{x}$ & & \\
\hline Cobolongo Thevetia nerifolia & $\mathrm{x}$ & $\mathrm{X}$ & $\mathrm{X}$ & $\mathrm{x}$ & $\mathrm{x}$ & & $\mathrm{x}$ \\
\hline Calistemo Calistemon citrinus & & & & & & & $\mathrm{x}$ \\
\hline Cipres Cupressus sp. & & & $\mathrm{X}$ & $\mathrm{X}$ & & & \\
\hline Estoraque Liquidambar styraciflua & & & & & & $\bar{x}$ & \\
\hline Eucalipto Eucalyptus sp. & $\mathrm{x}$ & $\mathrm{X}$ & & $\mathrm{x}$ & $\mathrm{x}$ & $\mathrm{x}$ & $\mathrm{X}$ \\
\hline Flamboyán Delonix regia & & & & & & & \\
\hline FicusFicus benjamina & $\mathrm{X}$ & $\mathrm{X}$ & & $\bar{x}$ & $\mathrm{X}$ & & $\bar{x}$ \\
\hline Limpia botellas Callistemon citrinus & & & & & & $\bar{x}$ & \\
\hline Lluvia de oro Cassia fistula & $\mathrm{X}$ & $\mathrm{x}$ & $\mathrm{X}$ & & $\mathrm{x}$ & & $\mathrm{X}$ \\
\hline Magnolio Magnolia grandiflora & & & & & & $\mathrm{x}$ & \\
\hline Mandarina Citrus reticulata & & $\mathrm{X}$ & & & & & \\
\hline Mango Manguifera indica & $\mathrm{X}$ & $\mathrm{X}$ & $\bar{x}$ & $\mathrm{x}$ & $\bar{x}$ & & $x$ \\
\hline Mión Spathodea campanulata & $\mathrm{x}$ & & & & & & \\
\hline Oití Licania tomentosa & $\mathrm{x}$ & $\mathrm{X}$ & & $\mathrm{x}$ & $\mathrm{x}$ & & $\mathrm{X}$ \\
\hline Palo santo Guaiacum sanctum & & $\mathrm{X}$ & & & & & \\
\hline Palma abanico Pritchardia pacifica & $\mathrm{X}$ & $\mathrm{X}$ & $\bar{x}$ & $\mathrm{x}$ & $\bar{x}$ & $\mathrm{x}$ & $x$ \\
\hline Palma africana Elaeis guianensis & & & $\mathrm{X}$ & & & & $\mathrm{X}$ \\
\hline Palma areca Crysalidocarpus lutescens & $\mathrm{x}$ & $\mathrm{X}$ & $\mathrm{X}$ & $\mathrm{x}$ & $\mathrm{x}$ & $\mathrm{x}$ & $\mathrm{X}$ \\
\hline Palma de azúcar Syagrus romanzoffiana & & & & & & & $\mathrm{X}$ \\
\hline Palma de coco Cocos nucifera & & & $\bar{x}$ & & $\bar{x}$ & & $\bar{x}$ \\
\hline Palma cola de pescado Caryota mitis & $\mathrm{X}$ & $\mathrm{X}$ & $\mathrm{x}$ & $\mathrm{x}$ & $\mathrm{X}$ & $\mathrm{x}$ & $\mathrm{X}$ \\
\hline Palma cubana Roystonea regia & & & & & & $\mathrm{x}$ & \\
\hline Palma de Manila Veitchia merrillii & $\mathrm{X}$ & $\mathrm{X}$ & $\mathrm{X}$ & $\mathrm{X}$ & $\mathrm{X}$ & $\bar{x}$ & $\mathrm{X}$ \\
\hline Palma payanesa Archontophoenix cunninghamiana & & & & & & $\mathrm{X}$ & \\
\hline Palma del viajero Ravenala madagascariensis & $\mathrm{X}$ & $\mathrm{X}$ & $\mathrm{X}$ & $\mathrm{X}$ & $\mathrm{x}$ & & $x$ \\
\hline Palma fénix Phoenix canariensis & $\mathrm{x}$ & $\mathrm{X}$ & $\mathrm{X}$ & & $\mathrm{x}$ & & \\
\hline Palma paraíso Veitcbia merrillii & & $\mathrm{X}$ & & & $\mathrm{x}$ & & \\
\hline Palma real Roystonea regia & $\mathrm{X}$ & $\mathrm{X}$ & $\mathrm{X}$ & $\mathrm{x}$ & $\mathrm{X}$ & & $\mathrm{X}$ \\
\hline Palma mariposa Caryota ureas & & & $\mathrm{X}$ & $\mathrm{x}$ & & & $\mathrm{x}$ \\
\hline Palma robeline Pboenix robellini & & & $\mathrm{X}$ & $\mathrm{X}$ & $\mathrm{x}$ & $\mathrm{x}$ & $\mathrm{X}$ \\
\hline Palma washingtoniana $W$ ashingtonia filifera & & & & & $\mathrm{x}$ & & \\
\hline Pomo rozo Syzygium malaccense & & & & $\mathrm{x}$ & & & \\
\hline Pino Pinus patula & & & $\bar{x}$ & $\mathrm{x}$ & & $\mathrm{x}$ & $x$ \\
\hline Pino Pinus radiata & & & $\mathrm{X}$ & $\mathrm{X}$ & & $\mathrm{x}$ & $\mathrm{X}$ \\
\hline Patevaca Baubinia variegata & $\mathrm{x}$ & $\mathrm{x}$ & & & $\mathrm{x}$ & $\mathrm{x}$ & $\mathrm{X}$ \\
\hline Pomarroso brasileño Syzygium malaccense & $\mathrm{x}$ & & $\mathrm{X}$ & & $\mathrm{X}$ & $\mathrm{x}$ & $\mathrm{X}$ \\
\hline Sauco Sambucus sp. & & & & & & & $\mathrm{x}$ \\
\hline Tamarindo Tamarindus indica & & & & $\mathrm{x}$ & & & \\
\hline Teca Tectona grandis & & & & & & & $\mathrm{X}$ \\
\hline Tulipán africano Spathodea campanulata & & $\mathrm{X}$ & $\mathrm{X}$ & $\bar{x}$ & $\bar{x}$ & $\bar{x}$ & $x$ \\
\hline Urapán Fraxinun cbinensis & & & $x$ & & & $\mathrm{X}$ & $\bar{x}$ \\
\hline
\end{tabular}


Es evidente que en las siete ciudades estudiadas los programas de arborización urbana han recurrido tanto a especies nativas como a introducidas, pero han sido las introducidas las que se han sembrado masivamente, generando verdaderos monocultivos urbanos con especies que no establecen muchas relaciones simbióticas con los entornos ecológicos locales (por ser oriundas de otras regiones del planeta). Aunque hace más de 30 años algunas de esas especies introducidas se vienen reportando como inadecuadas para los centros urbanos por sus raíces agresivas, que causan severos y graves daños en asfaltos, andenes, redes de infraestructura y edificaciones, estas especies se siguen plantando masivamente en muchas ciudades colombianas. Ejemplo de lo anterior es la ciudad de Villavicencio, donde, para un total de 11.668 árboles inventariados en el año 2000 (BERNAL, 2000), se reportaron 3.679 individuos de Ficus benjamina, es decir el $31.5 \%$ del total de árboles plantados en la ciudad; mientras que el pomo rozo Syzygium malaccense, reporta en el mismo estudio, un total de 3.725 individuos, es decir el 31\% de los árboles incluidos en la muestra. De manera que tan sólo esas dos especies introducidas, representan el 63\% de los árboles de la ciudad. La predominancia de las especies introducidas en la arborización urbana se aprecia en la relación entre las poblaciones de especies introducidas frente a las especies nativas, que en Villavicencio es de 10 a 1, respectivamente. Esta situación no es exclusiva de las ciudades colombianas, puesto que un estudio realizado en la ciudad de Ponta Grosa, Estado de Paraná, Brasil, reporta que:

"Foram percorridas 59 vias possibilitando a identificação de 479 indivíduos arbóreos, dos quais 27\% são espécies nativas e 73\% exóticas, sendo a espécie Lagerstroemia indica (extremosa) a espécie que mais se destacou com 18\%. Esse resultado demonstra a tendência nos centros urbanos da predominância de espécies exóticas e a fraca participação desse elemento verde na paisagem urbana, já que a média por via foi de 8,11 indivíduos arbóreos” (RODRIGUES; CARVALHO, 2010, p. 42).

Otro estudio, realizado en tres barrios de la ciudad de Pombal, Estado de Paraíba, Brasil, reporta que para un total de 212 árboles incluidos en la muestra, la especie predominante fue el Ficus benjamina, con el 51\% del total de individuos (RODOLFO JÚNIOR et al., 2008, p. 3).

\section{Especies no aptas para la arborización urbana}

Para establecer cuáles especies de las incluidas en la investigación no son aptas para la arborización urbana, se revisó la literatura científica así como la normativa especializada en el tema, con el objetivo de identificar las especies reportadas porque:

1. Generan riesgo directo o indirecto para la salud humana:

a) Por ser tóxicas:

-Cobolongo Thevetia nerifolia (CARVAJAL \& CHACÓN, 1999; COSTA SILVA, 2009 p. 83)

-Habillo Hura crepitans (TRUJILLO, 1997, p. 77)

b) Porque se autopodan, es decir, deja caer pesadas ramas generando altos riesgos de accidente en el espacio público; o se desploman, causando daños severos a personas, vehículos, construcciones arquitectónicas y civiles:

-Caracolí Anacardium excelsum (información suministrada por expertos del Jardín Botánico Eloy Valenzuela).

-Eucalipto Eucalipto glóbulus (en Bogotá se han identificado más de 900 individuos de esta especie en riesgo de desplome, y varios han caído, generando grandes daños).

c) Porque pueden generar accidentes de tránsito debido a sus grandes frutos:

-Algarrobo Hymenaea courbaril.

-Árbol del pan Artocarpus communis (VARÓN \& MORALES, 1997, p. 93).

d) Porque atraen plagas, generando riesgos de salud pública:

-Cacao Theobroma cacao (JARDÍN BOTÁNICO ELOY

VALENZUELA DE BUCARAMANGA, CONSEJO DE NEIVA, 2003, p. 7).

-Palma africana Elaeis guineensis (TRUJILLO, 1997, p. 179).

EXPEDIÇÃO BOTANICA URBANA EM... 
2. Porque su sistema radicular agresivo causa daños a la infraestructura vial y de servicios públicos, así como a construcciones arquitectónicas y civiles:

-Árbol del pan Artocarpus communis (CALDAS, 1975, p. 115; VARÓN \& MORALES, 1997, p. 93; HERRERA, 2009, p. 17).

-Caucho de la India Ficus elástica (KONYA, 1981, p. 135; CONSEJO MUNICIPAL DE IBAGUÉ, 1989, p. 5; CONSEJO DE NEIVA, 2003; VARÓN \& MORALES, 1997, p. 95; CALDAS, 1975, p. 80; IGAC, 1997, p. 63; CÁRDENAS et al, 2005, p. 58).

-Caucho lira Ficus lirata (KONYA, 1981, p. 135).

-Castañete Pachira acuatica (CALDAS, 1975, p. 124).

-Ficus Ficus benjamina (HERRERA, 2009, p. 138; CALDAS, 1975, p. 176; CONSEJO MUNICIPAL DE IBAGUÉ, 1989, p. 5; VARÓN \& MORALES, 1996, p. 95; BERNAL, 2000).

-Flamboyán Delonix regia (CALDAS, 1975, p. 83; CONSEJO MUNICIPAL DE IBAGUÉ, 1989, p. 5; CÁRDENAS et al, 2005, p. 50; HERRERA, 2009, p. 118).

-Tulipán africano Spathodea campanulata (LOWE et al, 2004, p. 6; CALDAS, 1975, p. 93;).

3. Porque resecan, erosionan o alteran la composición química del suelo, o producen hojarascas que taponan las redes de alcantarillado:

-Eucalipto Eucalipto globulus (GARCÍA BARRIGA, 1972, p. 273; CONSEJO MUNICIPAL DE IBAGUÉ, 1989).

-Pino de Monterrey Pinus patula (CONSEJO MUNICIPAL DE IBAGUÉ, 1989; MOLINA; GONZÁLEZ; SÁNCHEZ, 1995, p. 141).

-Pino candelabro Pinus radiata (CONSEJO MUNICIPAL DE IBAGUÉ, 1989; MOLINA; GONZÁLEZ; SANCHEZ, 1995, p. 142).

Algunas especies ornamentales introducidas que se caracterizan por sus raíces agresivas o porque son potencialmente riesgosas para la salud humana, aunque han sido reportadas por una amplia variedad de autores, se siguen plantando en las ciudades colombianas y de otros países. Esto genera elevados costos, no previstos en el momento de la plantación, que deben asumir tanto los contribuyentes como los propietarios de los bienes afectados (costos de reparación de infraestructura vial y de servicios públicos, costos por daños a vehículos, obras arquitectónicas y civiles, y costos humanos generados por accidentes en el espacio urbano). El estudio adelantado por Bernal (2000) en Villavicencio evidencia los daños generados por el arbolado urbano, pues reporta 3.478 afectaciones severas al acueducto y el alcantarillado, 196 afectaciones severas al alumbrado público, 963 afectaciones severas a las redes de energía y 1.072 afectaciones severas a las redes telefónicas, todas ellas generadas por individuos de Ficus benjamina. Asimismo, el estudio realizado por Costa Silva (2009), en la ciudad de Teresina, Estado de Piaui al norte del Brasil, da cuenta de una serie de especies potencialmente tóxicas plantadas en el espacio público estudiado.

\section{Selección de especies recomendadas}

Para la selección de especies (aptas para los ambientes urbanos) y que fortalecen la Estructura Ecológica Principal de las ciudades, se diseñó una matriz, en la que se tuvieron en cuenta las siguientes variables.

\section{Suelo:}

1. Nitrifica: especie que fija nutrientes al suelo (nitrógeno, sodio, potasio), al entrar en simbiosis con algunos microorganismos como las micorrizas.

2. Estructura: especie que proporciona estructura al suelo, reduciendo los riesgos de deslizamiento y minimizando los procesos de erosión hídrica.

3. Conserva: especie que colabora en la conservación del suelo.

4. Rehabilita: especie que contribuye en la rehabilitación y restauración ecológica de suelos erosionados o afectados por canteras.

Agua:

5. Conserva cuencas: especie que contribuye en la conservación de cuencas, microcuencas, rondas hídricas y nacederos de agua.

6. Rehabilita cuencas: especie que colabora en la rehabilitación ecológica de rondas y cuerpos de agua afectados por procesos erosivos o causas antrópicas.

Luis Fernando Molina-Prieto 
Fauna (atrae o alimenta):

7. Aves melíferas: especie productora de néctar y polen.

8. Aves semilleras: especie productora de semillas.

9. Aves frugívoras: especie productora de fruta.

10. Aves insectívoras: especie que atrae insectos, y las aves que de ellos se alimentan.

11. Loros: especie que forma parte de la alimentación de guacamayas, loros y pericos.

12. Rapaces: especie que atrae gavilanes, búhos o lechuzas.

13. Murciélagos: especies polinizada por estos mamíferos, o que produce fruta que ellos consumen.

14. Ardillas: especie que alimenta a estos mamíferos.

15. Iguanas, reptiles: especie que sirve de hábitat a los reptiles.

16. Abejas y otros: especie utilizada en apicultura.

\section{Ecosistema:}

17. Hospeda flora: especie sobre la que crecen plantas epífitas, como las orquídeas y las bromelias.

18. Hospeda fauna: especie que sirve de hábitat a diversas especies de aves, pequeños mamíferos, reptiles e insectos.

La matriz propuesta por la investigación se nutrió con los datos obtenidos durante los trabajos de campo en las siete ciudades, y con los suministrados por las Corporaciones Autónomas Regionales y los jardines botánicos locales, y se complementó con la información publicada por: Barón y Morales (1997), Bernal (2000), Caldas (1975), Carvajal y Chacón (1999), Cárdenas et al. (2005), Hilty y Brown (1986), Molina y Osorio (1995), Molina, González y Sánchez (1995), Rodríguez-Mahecha y HernándezCamacho (2002), Segovia et al. (2000), Álvarez (1987) además de datos obtenidos de la Revista Sig-Pafc (1997) y las páginas http://www.conabio.gob.mx, y http://www.fs.fed.us/.

Se seleccionaron y recomendaron las especies que cumplen con seis o más funciones ecológicas de las planteadas por la matriz, puesto que estas especies poseen mayor potencial para contribuir al fortalecimiento de la Estructura Ecológica Principal de las ciudades estudiadas, y se indicó cuáles lugares de la ciudad son adecuados para la correcta plantación de cada especie de acuerdo a su tamaño (parques, orejas de puentes, glorietas, separadores viales, rondas hídricas, andenes). Las especies recomendadas por la investigación y los beneficios ecológicos que desempeñan cada una de ellas se presentan en la Matriz para la selección, Tabla 3. 
Tabla 3. Matriz para la selección

Table 3. Selection matrix

\begin{tabular}{|c|c|c|c|c|c|c|c|c|c|c|c|c|c|c|c|c|c|c|}
\hline \multirow[b]{2}{*}{ ESPECIES NATIVAS } & \multicolumn{4}{|c|}{ SUELO } & \multicolumn{2}{|c|}{ AGUA } & \multicolumn{10}{|c|}{ FAUNA } & \multicolumn{2}{|c|}{ ECOSIS. } \\
\hline & $\begin{array}{l}\frac{\mathbb{U}}{\underline{\underline{u}}} \\
\underline{\underline{\underline{E}}} \\
\mathbf{z}\end{array}$ & 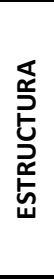 & 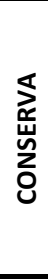 & 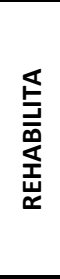 & 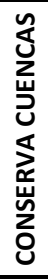 & 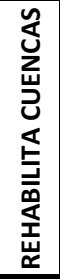 & 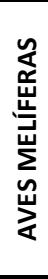 & 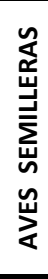 & 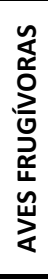 & 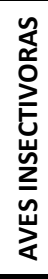 & 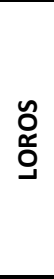 & 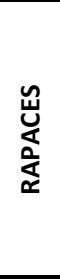 & 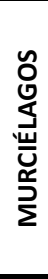 & $\begin{array}{l}\stackrel{y}{\bar{y}} \\
\stackrel{\bar{\alpha}}{\frac{\alpha}{\alpha}}\end{array}$ & 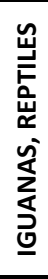 & 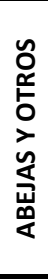 & 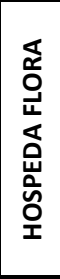 & 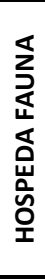 \\
\hline Acacia forrajera & $X$ & $x$ & $X$ & $x$ & & & & $\mathrm{X}$ & & $x$ & & & & & & $\mathrm{X}$ & & $x$ \\
\hline Aliso & $x$ & $x$ & $x$ & $x$ & $x$ & $\mathrm{X}$ & & & & & & & & & & & & $x$ \\
\hline Balso & & $x$ & $x$ & $\mathrm{X}$ & & & $x$ & $x$ & & $x$ & $x$ & & & & & $x$ & & $\mathrm{X}$ \\
\hline Búcaro & $x$ & $x$ & $x$ & $x$ & $x$ & $x$ & $x$ & & & $x$ & $x$ & $X$ & $x$ & & $x$ & $x$ & $x$ & $x$ \\
\hline Carbonero & $x$ & $x$ & $x$ & $\mathrm{X}$ & $x$ & $x$ & $x$ & & & $x$ & & & & & & $x$ & & \\
\hline Carbonero rojo & & & & & & & $x$ & & & & & & & & & $x$ & & $x$ \\
\hline Cachimbo & $x$ & $x$ & $x$ & $\mathrm{X}$ & $x$ & $x$ & $x$ & & & $x$ & $x$ & $x$ & $x$ & & $x$ & $x$ & $\mathrm{X}$ & $x$ \\
\hline Chachafruto & $x$ & $x$ & $x$ & $x$ & $x$ & $x$ & $x$ & & & $x$ & $x$ & $x$ & $x$ & & $x$ & $x$ & $\mathrm{X}$ & $x$ \\
\hline Ceiba & $\mathrm{X}$ & $x$ & $\mathrm{X}$ & $\mathrm{X}$ & & & $\mathrm{X}$ & & & $\mathrm{X}$ & $x$ & & $x$ & & & $\mathrm{X}$ & $x$ & $x$ \\
\hline Cují & $x$ & $x$ & $x$ & $\mathrm{X}$ & & & $x$ & & & & & & $x$ & & & $x$ & & $x$ \\
\hline Gallinero & $x$ & $x$ & $x$ & $\mathrm{X}$ & & & $x$ & $x$ & & $x$ & $x$ & & $x$ & & & $x$ & $\mathrm{X}$ & $x$ \\
\hline Guadua & & & $x$ & & $x$ & $\mathrm{X}$ & & $x$ & & & $x$ & & & & & & & $x$ \\
\hline Guásimo & & $x$ & $x$ & $\mathrm{X}$ & & & & & & & $x$ & & & & & $\mathrm{x}$ & & $x$ \\
\hline Guamo & $\mathrm{X}$ & $x$ & $\mathrm{X}$ & $\mathrm{X}$ & & & $\mathrm{X}$ & & & $\mathrm{x}$ & $x$ & & $x$ & & & $\mathrm{X}$ & & $x$ \\
\hline Guayabo & & $x$ & $x$ & $x$ & & & & & & & $x$ & & & & & $x$ & & $x$ \\
\hline Guayacán flor amarillo & $x$ & $x$ & $x$ & $\mathrm{X}$ & & & $\mathrm{x}$ & & & $\mathrm{x}$ & $x$ & & $x$ & & & $\mathrm{x}$ & & $x$ \\
\hline Guayacán rosado & $x$ & $x$ & $x$ & $\mathrm{X}$ & & & $x$ & & $x$ & $x$ & $x$ & & $x$ & & & $x$ & & \\
\hline Iguá & $x$ & $x$ & $x$ & $x$ & & & $x$ & & & $x$ & $x$ & & $x$ & & & $x$ & & $x$ \\
\hline Mamón & & & & & & & $x$ & & $x$ & $x$ & $x$ & & $x$ & & & $x$ & & $x$ \\
\hline Mango & & & & & & & $x$ & & $x$ & $x$ & $x$ & $x$ & $x$ & & & $x$ & & $x$ \\
\hline Mano de tigre & & $x$ & $x$ & $\mathrm{X}$ & & & $\mathrm{x}$ & $\mathrm{x}$ & & $\mathrm{x}$ & $x$ & $x$ & $x$ & $x$ & $\mathrm{x}$ & $\mathrm{x}$ & $\mathrm{X}$ & $x$ \\
\hline Matarratón & $x$ & $x$ & $x$ & $x$ & $x$ & $x$ & $x$ & $\mathrm{x}$ & & $\mathrm{x}$ & $x$ & & $\mathrm{x}$ & & & $\mathrm{x}$ & & $x$ \\
\hline Orejero & $x$ & $x$ & $x$ & $x$ & & & & & & & & $x$ & & & & $x$ & & $x$ \\
\hline Nacedero & & & $\mathrm{X}$ & $\mathrm{X}$ & $x$ & $\mathrm{X}$ & $\mathrm{X}$ & & & $\mathrm{X}$ & & & & $x$ & & $\mathrm{X}$ & & $x$ \\
\hline Níspero & & $x$ & $x$ & $\mathrm{X}$ & & & $x$ & & $x$ & $x$ & & & $x$ & $x$ & & $\mathrm{x}$ & & $x$ \\
\hline Orejero & $x$ & $x$ & $x$ & $x$ & & & & & & & & $x$ & & & & $x$ & & $x$ \\
\hline Palo de cruz & & & & & $x$ & $x$ & $x$ & $x$ & & & & & $x$ & & & $x$ & & $x$ \\
\hline Samán & $\mathrm{X}$ & $x$ & $x$ & $\mathrm{X}$ & & & $x$ & & & $x$ & $\mathrm{x}$ & $x$ & $x$ & $x$ & $x$ & $\mathrm{x}$ & $\mathrm{X}$ & $x$ \\
\hline Totumo & & $x$ & $x$ & $X$ & & & $x$ & & & $x$ & $x$ & & $x$ & & & $x$ & & $x$ \\
\hline Yarumo & & & $x$ & & & & $x$ & $x$ & $x$ & $x$ & $x$ & & $x$ & & & $x$ & & \\
\hline Zapote & & & & & $x$ & $x$ & $x$ & & $x$ & $x$ & $x$ & & $x$ & & & $x$ & & $x$ \\
\hline
\end{tabular}

\section{CONCLUSIONES}

La ausencia de políticas claras en relación a las especies apropiadas para la arborización urbana de las ciudades colombianas (y en otros países como Brasil), genera afectaciones ecológicas, económicas y de salud pública.
Durante los últimos treinta años la literatura científica ha hecho énfasis en los daños y afectaciones que causan algunas especies en las ciudades, sin embargo, tanto arquitectos como urbanistas y planificadores urbanos, no Luis Fernando Molina-Prieto 
parecen tener en cuenta estas investigaciones, y en consecuencia, continúan plantando las especies más inadecuadas en sus proyectos, bien sean esos proyectos de pequeña, mediana o gran escala. Mientras se sigan plantando árboles, desconociendo sus efectos a mediano y largo plazo, se seguirán generando gastos innecesarios y poniendo en riesgo a los habitantes, en ciudades y municipios que en general cuentan con estrechos presupuestos económicos.

Las especies recomendadas por la investigación, fortalecen la Estructura Ecológica Principal de las siete ciudades incluidas en el estudio, y por tanto desempeñan funciones no sólo paisajísticas y ambientales, como el ornamento urbano, el bienestar psicológico de los habitantes y la reducción de la contaminación urbana, sino que además, proporcionan a las ciudades beneficios ecológicos que redundan en el bienestar de los ciudadanos, pues reducen los riesgos de deslaves o remociones en masa, regulan los caudales de quebradas, ríos y otros cuerpos de agua, beneficios que reducen los riesgos de catástrofes durante las épocas de invierno o alta pluviosidad; al tiempo que ofrecen alimento y hábitat a la fauna urbana, especialmente a las aves, tanto residentes como migratorias, contribuyendo con su conservación.

\section{REFERENCIAS BIBLIOGRÁFICAS}

ÁLVAREZ, H. Introducción a las aves de Colombia, segunda edición. Bogotá: Banco Popular, 1987. 200 p.

BARÓN P. T.; MORALES, S. L. Árboles del Valle de Aburrá. Medellín: Editorial Colina, 1997. 176 p.

BERNAL, C. A. Estudio diagnóstico y formulación de solución a las afectaciones de la flora urbana sobre la infraestructura vial y de servicios públicos-Municipio de Villavicencio. Villavicencio: Secretaría de Planeación, Secretaría del Medio Ambiente, 2000. 180 p.

CALDAS L. La flora ornamental tropical y el espacio urbano. Cespedesia, boletín científico del Departamento del Valle del Cauca, Cali, v. IV, n 14, p. 55-179, abril-junio, 1975.

CÁRDENAS, D.; ARIAS, J. C.: LÓPEZ, R. Árboles y arbustos de la ciudad de Leticia. Bogotá: SINCHI, 2004. 120 p.

CARVAJAL, E.; CHACÓN, J. Cúcuta verde y exuberante. Cúcuta: CORPONOR, 1999. 160 p.

CONSEJO MUNICIPAL DE IBAGUÉ. Estatuto del árbol urbano. Ibagué: Consejo Municipal de Ibagué, 1989. 8 p.

CONSEJO DE NEIVA. Acuerdo 044 de 2003 "Por medio del cual se reglamentan las competencias en materia de arborización y manejo silvicultural en el espacio público de la ciudad de Neiva, Huila. Neiva: Consejo de Neiva, 2003. 10 p.

COSTA SILVA, L. Plantas ornamentais tóxicas presentes no shopping Riverside Walk em Teresina - PI. Revista da Sociedade Brasileira de Arborização Urbana REVSBAU, Piracicaba - SP, v. 4, n. 3, p. 69-85, Set., 2009.

DAMA-CONIF. Guía para la plantación de árboles. Bogotá: DAMA-CONIF, 1999. 80 p.

DELGADO, V. M. Especies ornamentales usadas en áreas urbanas “Experiencia programa hojas verdes”. Bogotá: Cámara de Comercio de Bogotá, 1995. 80 p.

JARDÍN BOTÁNICO DE BOGOTÁ JOSÉ CELESTINO MUTIS-JBB. Manual verde. Bogotá: Jardín Botánico de Bogotá José Celestino Mutis, 1999, $32 \mathrm{p}$.

GARCÍA BARRIGA, H. Árboles de la Sabana de Bogotá. Revista de la Academia Colombiana de Ciencias Exactas, Físicas y Naturales, Bogotá, v. 13, n. 50, p. 273-277, 1972.

HERRERA, S. Árboles de la Universidad del Valle. Cali: Programa Editorial Universidad del Valle, 2009. 336 p.

HILTY S.; BROWN, W. A guide to the birds of Colombia. New Jersey: Princeton University Press, 1986. 836 p.

JIMÉNEZ, O. Índice de confort de la vegetación. Revista Nodo, Bogotá, v. 3, n. 5 p. 49-70, julio-diciembre, 2008.

KONYA, A. Diseño en climas cálidos. Madrid: H. Blume Ediciones, 1980. 156 p.

LOWE, S. et al. 100 de las especies exóticas invasoras más dañinas del mundo. Auckland: GEEI, UICN, ISSG, 2004. 12 p.

MOLINA L.F.; GONZÁLEZ, M.; SÁNCHEZ, G. Guía de Árboles de Santafé de Bogotá. Bogotá: DAMA, 1995. 168 p. 
MOLINA L. F.; OSORIO, J. Guía de aves de Santafé de Bogotá. Bogotá: DAMA, 1995. 228 p.

MOLINA, L. F. Arborizaciones urbanas en clima cálido. Revista Nodo, Bogotá, v. 1, n. 2 p. 13-24, enero-junio, 2007.

PUCCINI, E. Arborizaciones en el Distrito de Barranquilla. Barranquilla: DADIMA, 2001.

RODRIGUES DE OLIVEIRA, A. C.; CARVALHO, S. C. Arborização de vias públicas e aspectos sócio-econômicos de três vilas de Ponta Grossa, PR. En Revista da Sociedade Brasileira de Arborização Urbana REVSBAU, Piracicaba - SP, v. 5, n. 3, p. 42-58, Set., 2010.

RODRÍGUEZ-MAHECHA J.; HERNÁNDEZ-CAMACHO, J. Loros de Colombia. Bogotá: Conservación Internacional, 2002. $480 \mathrm{p}$.

ROMERO, C. F. Masas forestales en cinco parques de Neiva. Revista Nodo, Bogotá, v. 3, n. 5, p. 85-99, julio-diciembre, 2008.

SECRETARÍA DEL MEDIO AMIENTE/JARDÍN BOTÁNICO DE MEDELLÍN. Manual de silvicultura urbana para Medellín. Medellín: Secretaría del Medio Amiente/Jardín Botánico de Medellín, 2007. 158 p.

SEGOVIA, R. J. et al. Árboles, arbustos y aves en el agrosistema del CIAT. Cali: Centro Internacional de Agricultura Tropical, 2000. 56 p.

TRUJILLO, E. Requerimientos, limitaciones y usos de especies forestales en Colombia. Revista informativa del proyecto SIG-PAFC, Bogotá, año 4, n. 14, p. 8-235, Sep., 1997.

WIESNER, D. Metodología para la definición de la estrategia de arborización. Memorias del foro de arborización urbana, Bogotá. Bogotá: Alcaldía Mayor de Bogotá, 2000. pp. 18- 29.

http://www.conabio.gob.mx

http://www.fs.fed.us 